\begin{abstract}
Frequency-domain analysis has been successfully used to (i) predict the amplification of traffic oscillations along a platoon of vehicles with nonlinear car-following laws and (ii) measure traffic oscillation properties (e.g., periodicity, magnitude) from field data. This paper proposes a new method to calibrate nonlinear car-following laws based on real-world vehicle trajectories, such that oscillation prediction (based on the calibrated car-following laws) and measurement from the same data can be compared and validated. This calibration method, for the first time, takes into account not only the driver's car-following behavior but also the vehicle trajectory's time-domain (e.g., location, speed) and frequency-domain properties (e.g., peak oscillation amplitude). We use Newell's car-following model (1961) as an example and calibrate its parameters based on a penalty-based maximum likelihood estimation procedure. A series of experiments using Next Generation Simulation (NGSIM) data are conducted to illustrate the applicability and performance of the proposed approach. Results show that the calibrated car-following models are able to simultaneously reproduce observed driver behavior, time-domain trajectories, and oscillation propagation along the platoon with reasonable accuracy.
\end{abstract}

(C) 2016. This manuscript version is made available under the Elsevier user license http://www.elsevier.com/open-access/userlicense/1.0/ 
Keywords: Nonlinear Car-Following Law, Traffic Oscillation, Model Calibration 


\title{
Calibration of Nonlinear Car-Following Laws for Traffic Oscillation Prediction
}

\author{
Christine Rhoades ${ }^{1}$, Xin Wang ${ }^{2}$, Yanfeng Ouyang*1 \\ ${ }^{1}$ Department of Civil and Environmental Engineering, University of Illinois, \\ Urbana-Champaign \\ ${ }^{2}$ Department of Industrial and Systems Engineering, University of Wisconsin-Madison
}

\section{Introduction}

Drivers in congested traffic find it extremely difficult to maintain a steady speed; instead, they often engage in frequent acceleration-deceleration cycles - a phenomenon commonly referred to as "stop-and-go traffic" or "traffic oscillation". This phenomenon causes a range of problems including safety hazards, extra fuel consumption, extra emissions, travel delay, and driver discomfort. For decades, researchers have been developing theoretical and empirical methods to better understand the mechanism of the traffic oscillation phenomenon and to seek solutions to mitigate its adverse effects.

Theoretical efforts to study traffic oscillation can be traced back to the 1950's (Chandler et al., 1958; Herman et al., 1959). The main goal of these efforts is to reveal root causes of traffic oscillation in a platoon and quantify the impacts of individual driver's car-following behavior on its evolution. In congested traffic flow, a platoon of vehicles can be modeled as cascading dynamic systems, each consisting of a pair of consecutive vehicles with a certain car-following law describing the driver's behavior (Chandler et al., 1958). Each vehicle trajectory can be considered to consist of two components: a nominal component that describes the macroscopic characteristics (e.g., flow, density, average speed) and an oscillation component that describes the microscopic characteristics (e.g., oscillation period and amplitude) ( $\mathrm{Li}$ et al., 2010). To accurately model a platoon, a good car-following law must reproduce both of these components, and yet, accuracy in the oscillation component is essential for studying traffic oscillation. While the nominal component can be naturally studied in the time domain, it is generally convenient to analyze the oscillation component in the frequency domain. For example, earlier analyses of linear car-following models by frequencydomain techniques yielded insightful closed-form analytical results (Herman et al., 1959). A wellknown problem of linear analysis, however, is that the predicted oscillation amplitude would grow exponentially as the oscillation propagates along the platoon. This result obviously contradicts the bounded amplitude growth that we observe in reality (e.g., vehicles do not travel backwards, and they do not collide). Some researchers believe that the inaccuracy associated with the linear models

${ }^{*}$ Corresponding author, email: yfouyang@illinois.edu. 
probably lies in the lack of physical operational bounds, such as lower and upper acceleration/speed limits for a driver. Various nonlinear car-following laws have been developed to more accurately explain and reproduce traffic oscillation propagation (Gazis et al., 1961; Gipps, 1981; Bando et al., 1995; Treiber et al., 2000). For example, Newell's parsimonious piecewise linear car-following law (Newell, 1961) reproduces traffic evolution quite well while using only a few parameters. Such nonlinear models, however, are typically much more difficult to analyze in closed forms, and they often are studied via numerical simulations. Just recently, Li and Ouyang (2011) made some progress in overcoming this challenge by developing a describing function approach (DFA) framework to analytically characterize oscillation propagation properties under a general class of nonlinear carfollowing law.

Empirical studies on traffic oscillation were traditionally conducted on aggregated traffic data, e.g., those from loop detectors (Koshi et al., 1983; Ferrari, 1989; Kuhne, 1987; Zielke et al., 2008). Recently, availability of microscopic data such as vehicle trajectories provided the opportunity for more detailed observation and analysis. Most work thus far, however, has only measured traffic oscillation characteristics in the time domain. For example, Neubert et al. (1999) conducted a statistical analysis based on a single vehicle trajectory in the time-space diagram. Treiber and Helbing (2002) implemented data fusion methods to extract traffic state information from a spatiotemporal traffic profile consisting of a platoon of vehicle trajectories. Laval (2011) proposed a method based on kinematic wave theory to measure traffic flow variables. Recently, the advantages of applying frequency-domain analysis techniques to obtain more accurate measurements of oscillation characteristics have become apparent. Li et al. (2010) proposed a short-time Fourier transform method to measure oscillation properties. This framework was later extended to wavelet analysis methods (Zheng et al., 2011) and an extended spectral envelope method (Zhao et al., 2014).

Given these findings and insights of traffic oscillations based on various car-following laws, it would be ideal to validate the theoretical predictions with field observations. The availability of detailed microscopic traffic trajectory data significantly helps achieve this goal in two aspects. First, such data could be used to calibrate suitable car-following models, which can then be used as the basis for theoretical prediction of oscillations. Second, traffic oscillation properties could be properly measured from the same field data for comparison with the theoretical predictions, and in so doing serve as the benchmark for cross validation. In this way, both the theoretical predictions and the field measurements are validated, and the oscillation mechanism can be better understood. However, calibrating a car-following law is not trivial, especially when the car-following model is nonlinear, since both time-domain (such as location, speed, and acceleration) and frequency-domain properties (such as oscillation amplitude and periodicity) should be considered. Limited attempts have been made to quantitatively develop a suitable nonlinear car-following model that can closely match with field data. Most validation attempts have focused only on finding models that can reproduce timedomain properties (Kesting and Treiber, 2008; Treiber and Kesting, 2012). For example, Ciuffo et al. (2007) propose a calibration framework integrating model sensitivity analysis and validate their results via simulation.

Such models tend to ignore frequency-domain properties and normally yield inaccurate predictions of oscillation propagation. In light of this, recent studies begin to focus on the possibility of calibrating oscillation properties from the field data. Several efforts have been made through calibrating parsimonious models, which normally consist of a simple car-following law sharing by all vehicles as a base and certain driver specific parameters to partially capture the heterogeneity. In this stream, Chen et al. (2012) calibrate a specific behavioral car-following law based on vehicle 
trajectories to study the oscillation. Laval et al. (2014) further develop a stochastic model to relate the oscillation to the uncertainty when vehicle accelerates. These models, although only considering time-domain information, can generate oscillation propagation from a macroscopic perspective. To accurately reproduce the oscillation propagation, frequency-domain tools have to be implemented directly in the calibration. In light of this, Li et al. (2012) proposed a way to calibrate a car-following model from field trajectory data with extracted frequency-domain characteristics. However, there are two issues limiting its applicable field in practice: the shortage of time-domain traffic characteristics and the homogeneous driver's behavior setting in the calibration process. As a result, while this method yields a rather accurate reproduction of oscillation propagation in average, it fails to match the prediction of time-domain trends in vehicle trajectories and the details of stepwise oscillation propagation. As such, a new method is needed - one that can calibrate car-following laws for a platoon of heterogeneous drivers and properly reproduce both time- and frequency-domain properties.

To fill this gap, this paper proposes a new framework that calibrates the parameters of nonlinear car-following laws using field vehicle trajectories. Our framework focuses on calibrating the carfollowing law for each pair of consecutive vehicles, while explicitly incorporating frequency domain characteristics. Through a simulation algorithm, for the first time, the calibrated laws are capable of reproducing oscillation properties such as periodicity and magnitude, as well as location and speed profiles of vehicle trajectories. In addition, we obtain theoretical prediction from DFA as cross-validation to the simulation results. In particular, we conduct the calibration through a combination of maximum likelihood estimation and minimization of feedback penalties associated with both time- (trajectory) and frequency- (oscillation) domain prediction errors. The prediction errors in the time and frequency domains respectively capture the differences between the simulated (based on the calibrated car-following law) and the actual trajectories, and the differences between the simulated and the measured peak oscillation amplitudes. A series of numerical experiments based on NGSIM data ${ }^{1}$ are conducted to illustrate the satisfactory performance of the proposed approach by calibrating the Newell's car-following law as an example.

The rest of the paper is organized as follows. First, Section 2 introduces the model calibration framework. Section 2.1 proposes a maximum likelihood estimation to calibrate a general nonlinear car-following law; building upon this, Section 2.2 defines both time- and frequency-domain errors and uses them to achieve better traffic reproduction. Section 2.3 describes the solution technique used to calibrate the model. Section 3 describes the DFA method that can be used for analytical oscillation propagation prediction. Then, a series of numerical experiments, which validate the framework, are conducted in Section 4. Finally, Section 5 concludes the paper and discusses possible future research directions.

\section{Methodology}

We start this section by describing a general model calibration technique based on velocity and spacing observations. Then, a new feedback mechanism is introduced to improve traffic reproduction accuracy in time- and frequency-domains.

\footnotetext{
${ }^{1}$ Source: http://ngsim.fhwa.dot.gov/
} 


\subsection{Model Calibration}

We consider a field trajectories set from a platoon of $n+1$ vehicles in one lane, indexed from the downstream to the upstream by $i=0,1, \ldots, n$. For vehicle $i$, let $x_{i}(t)$ be the recorded position at time $t \in T_{i}$, where $T_{i}$ is the discrete recorded time set with sampling step length $\Delta t$ (e.g., $T_{i}=\{0 \mathrm{~s}$, $0.1 \mathrm{~s}, 0.2 \mathrm{~s}, \ldots\})$, and $\mathbf{x}_{i}=\left\{x_{i}(t)\right\}_{t \in T_{i}}$ be the entire trajectory, assumed to be monotonically increasing over $t$.

For a given pair of consecutive trajectories $\left\{\mathbf{x}_{i-1}, \mathbf{x}_{i}\right\}$, at time $t$, the observed velocity $v_{i}(t)$ of the following vehicle is estimated from data as

$$
v_{i}(t) \approx \frac{x_{i}(t+\Delta t)-x_{i}(t)}{\Delta t}
$$

and the observed spacing by driver $i, s_{i}\left(t, \tau_{i}\right)$, is a time shift of $x_{i-1}(t)-x_{i}(t)$, i.e.,

$$
s_{i}\left(t, \tau_{i}\right)=x_{i-1}\left(t-\tau_{i}\right)-x_{i}\left(t-\tau_{i}\right) .
$$

assuming a time lag of $\tau_{i}$, which can, for example, account for reaction time.

For simplicity, we assume each vehicle independently follows a general class of nonlinear carfollowing law $F(\cdot)$ where the desired velocity of the following vehicle, $v_{i}^{*}(t)$, is defined using the spacing between the leading and following vehicles, $s_{i}\left(t, \tau_{i}\right)$, although the methodology suggested here could be modified to accommodate other types of laws as well. Each nonlinear car-following law can be specified by a parameter set $H_{i}$ in addition to $\tau_{i}$; i.e.,

$$
v_{i}^{*}(t)=\nu\left(s_{i}\left(t, \tau_{i}\right) \mid H_{i}\right) .
$$

We suppose that each observed velocity comes from the desired velocity plus an error term $\varepsilon_{i}$, as shown below:

$$
v_{i}(t)=v_{i}^{*}(t)+\varepsilon_{i}=\nu\left(s_{i}\left(t, \tau_{i}\right) \mid H_{i}\right)+\varepsilon_{i},
$$

where the error term $\varepsilon_{i}$ follows a general probability density function $\phi\left(\cdot \mid \Sigma_{i}\right)$ with its parameter set $\Sigma_{i}$

Given the trajectory data of the whole platoon $\left\{\mathbf{x}_{i}\right\}_{i=0}^{n}$, normally exhibiting congestion, we aim to obtain parameters $\left\{H_{i}, \tau_{i}, \Sigma_{i}\right\}_{i=1}^{n}$, each of which is calibrated based on a pair of consecutive trajectories; e.g., $\left\{\mathbf{x}_{i-1}, \mathbf{x}_{i}\right\}$. These calibrated parameters should reproduce $\left\{\mathbf{x}_{i}\right\}_{i=1}^{n}$ in both the time-space diagram (with regard to the trajectories) and the frequency-domain (with regard to the oscillation amplitudes).

The parameters can be calibrated by maximizing the likelihood function for observing any pair of trajectories $\left\{\mathbf{x}_{i-1}, \mathbf{x}_{i}\right\}$; i.e.,

$$
\mathcal{L}\left(H_{i}, \tau_{i}, \Sigma_{i}\right)=\prod_{t \in T_{i}} \phi\left(v_{i}(t)-\nu\left(s_{i}\left(t, \tau_{i}\right) \mid H_{i}\right) \mid \Sigma_{i}\right) .
$$

For notation convenience, we simply define $M_{i}=\left\{H_{i}, \tau_{i}\right\}$ to be the car-following law parameter set. Then, an open-loop calibration of the car-following law can be written as follows.

Maximum Likelihood Estimation (MLE):

$$
\left[M_{i}^{L}, \Sigma_{i}^{L}\right]=\underset{M_{i}, \Sigma_{i}}{\arg \max } \mathcal{L}\left(M_{i}, \Sigma_{i}\right)
$$


Note that $M_{i}^{L}$ contains the optimal parameters in the car-following law that will maximize the accuracy of predicting $v_{i}^{*}(t)$. This simple method has several limitations, however. First, the maximum likelihood function is highly nonlinear in the parameters, and hence the search for the best parameter values may easily become trapped in local optima. For example, the value of $H_{i}^{L}$ would be very sensitive to the value of reaction time $\tau_{i}^{L}$, as illustrated by the data plots in Figure 1. Second, the error terms are assumed to be independent; hence the estimation method above does not explicitly address the temporal continuity and autocorrelation of a driver's carfollowing behavior. Third, there is no explicit consideration of oscillation properties, and hence the resulting car-following law will be unlikely to accurately reproduce the oscillatory components (which will be illustrated later in our numerical experiments). Finally, the MLE is not likely to provide accurate calibration results in the case of skewed trajectories, e.g., where only a small portion of the trajectory exhibits an oscillation. Since the MLE gives equal weight to each velocityspacing sample point, the performance is likely to be dominated by the proportion of the trajectory without oscillation, while the oscillation can be extremely underestimated or even missing under reproduction. In the following subsections, we propose to improve the model parameter estimation by explicitly addressing feedbacks from the reproduction of both the time- and frequency-domain traffic components.

Place Figure 1 about here.

\subsection{Simulation-Based Feedback}

In this section, we will first introduce time- and frequency-domain traffic prediction errors. These errors will be used to evaluate any given car-following law $M_{i}$ and will later be incorporated into the calibration procedure via a penalty method.

\subsubsection{Time-Domain Feedback}

Since almost all field trajectory data are collected in the time domain, verifying $M_{i}$ in the time domain is intuitive. We only need to reproduce the trajectory of the following vehicle in the timespace diagram and measure the difference from its observed counterpart.

Given a pair of field trajectories $\left\{\mathbf{x}_{i-1}, \mathbf{x}_{i}\right\}$ that span time ranges $T_{i-1}$ and $T_{i}$, respectively, and a car-following model $M_{i}$, we simulate the following vehicle's trajectory $\hat{\mathbf{x}}_{i}$ as follows.

For any $t \in T_{i}$, if $t-\tau_{i} \notin T_{i-1} \cap T_{i}$, we have $\hat{x}_{i}(t)=x_{i}(t)$. Otherwise,

$$
\hat{x}_{i}(t+\Delta t)=\hat{x}_{i}(t)+\hat{v}_{i}(t) \Delta t,
$$

where $\hat{v}_{i}(t)=\nu\left(\hat{x}_{i-1}\left(t-\tau_{i}\right)-\hat{x}_{i}\left(t-\tau_{i}\right) \mid H_{i}\right)$ is the simulated velocity, as given by the car-following model.

Then, we define the time-domain error associated with parameters $M_{i}$ as

$$
e_{i}^{T}\left(M_{i}\right):=\frac{1}{\left|T_{i}\right|} \sqrt{\sum_{t \in T_{i}}\left[x_{i}(t)-\hat{x}_{i}(t)\right]^{2}},
$$

where $\left|T_{i}\right|$ is the number of elements in $T_{i}$. Obviously, a smaller $e_{i}^{T}$ indicates a more accurate reproduction of the time-domain trajectory. This error reflects on the accuracy of trajectory reproduction at every time step and hence addresses the continuity and autocorrelation of a driver's car-following decisions. 


\subsubsection{Frequency-Domain Feedback}

Most oscillation properties, e.g., amplitude and periodicity of oscillation, can be easily captured by analyzing the oscillation component $x_{i}^{o}(t)$ of the vehicle trajectory in the frequency-domain, which is defined here as in Li and Ouyang (2011).

First, we transform the time-domain field data into the frequency-domain. In the field data capturing congested traffic, especially when the "stop-and-go" phenomenon shows periodically in the trajectory, the frequency spectrum of $x_{i}^{o}(t)$ normally contains a highly dominant frequency component ( $\mathrm{Li}$ et al., 2010), i.e., the fundamental frequency, denoted by $\Omega_{i}$, and hence $x_{i}^{o}(t)$ can be approximated by a sinusoid, shifted to zero phase without loss of generality,

$$
x_{i}^{o}(t) \approx A_{i} \sin \left(\Omega_{i} t\right), \forall i \in T_{i},
$$

where $A_{i}$ is the oscillation amplitude. Considering that the field data are noisy and may only contain a small number of oscillation periods (e.g., only one stop-and-go cycle), which means the typical discrete Fourier transform is not effective, we use the following wavelet method (Daubechies, 1992) to estimate $\Omega_{i}$ and $A_{i}$ for vehicle $i$ 's trajectory. First, we estimate

$$
\Omega_{i}=\underset{\Omega}{\arg \max }\left\{\max _{t \in T_{i}} \int_{-\pi / \Omega}^{\pi / \Omega} \psi(u, \Omega) x_{i}^{o}(t-u) d u\right\},
$$

where

$$
\psi(u, \Omega)=\frac{\Omega}{\pi} \sin (\Omega u), \forall \Omega>0, u \in \mathbb{R},
$$

is the wavelet function. Let

$$
t_{i}^{m}=\arg \max _{t \in T_{i}} \int_{-\pi / \Omega_{i}}^{\pi / \Omega_{i}} \psi\left(u, \Omega_{i}\right) x_{i}^{o}(t-u) d u .
$$

Then, the observed oscillation amplitude, $A_{i}$, is estimated as

$$
A_{i}=\frac{\Omega_{i}}{\pi} \int_{t_{i}^{m}-\pi / \Omega_{i}}^{t_{i}^{m}+\pi / \Omega_{i}} e^{-j \Omega_{i} t} x_{i}^{o}(t) d t
$$

where $j$ is the imaginary unit.

We similarly apply the wavelet method described above to the simulated trajectory, denoted by $\hat{A}_{i}$. Then the frequency-domain error under $M_{i}$ can be defined using the maximum value on the trajectory's frequency spectrum (i.e., the one dictating the oscillation amplitude) as follows:

$$
e_{i}^{F}\left(M_{i}\right):=\left|A_{i}-\hat{A}_{i}\right| .
$$

Note that the periodicity information is not explicitly captured in the frequency-domain error, since the leading and the following vehicle normally share the same periodicity in oscillation.

\subsection{Model Integration and Solution}

The aforementioned MLE model provides a good starting point for finding the optimal parameters for the car-following law, but it can be further improved by incorporating the time- and frequencydomain errors. 
First, define

$$
\mathcal{M}_{i}\left(\gamma^{F}, \gamma^{T}\right):=\left\{M_{i}: e_{i}^{F}\left(M_{i}\right) \leq \gamma^{F}, e_{i}^{T}\left(M_{i}\right) \leq \gamma^{T}\right\}, i=1,2, \ldots, n,
$$

where $\gamma^{F}, \gamma^{T} \in \mathbb{R}_{+}^{2}$ are predetermined tolerances for the frequency- and time-domain errors. All parameters in $\mathcal{M}_{i}\left(\gamma^{F}, \gamma^{T}\right)$ guarantee acceptable traffic reproduction errors no larger than $\gamma^{F}$ and $\gamma^{T}$.

We assume that the model calibration shall be subject to guaranteed reproduction errors, as the following:

$$
\max _{M_{i} \in \mathcal{M}_{i}\left(\gamma^{F}, \gamma^{T}\right), \Sigma_{i}} \mathcal{L}\left(M_{i}, \Sigma_{i}\right), \text { if } \mathcal{M}_{i}\left(\gamma^{F}, \gamma^{T}\right) \neq \emptyset .
$$

It is obvious that $\left|\mathcal{M}_{i}\left(\gamma^{F}, \gamma^{T}\right)\right|$ is non-decreasing over either $\gamma^{F}$ or $\gamma^{T}$, and $\mathcal{M}_{i}(+\infty,+\infty) \neq \emptyset$. Further, if $\mathcal{M}_{i}(0,0) \neq \emptyset$, then (2.3) yields the ideal model that perfectly reproduces the field data in both the time and frequency domains. It is difficult, however, to judge if $\mathcal{M}_{i}\left(\gamma^{F}, \gamma^{T}\right) \neq \emptyset$ or to choose appropriate $\gamma^{F}$ and $\gamma^{T}$ from the field data before we solve (2.3).

To find a proper balance between the time- and frequency-domain errors without explicitly specifying the error thresholds, we propose the following penalty-based optimization (Yeniay, 2005)

Penalty-based Maximum Likelihood Estimation (pMLE):

$$
\lim _{p \rightarrow \infty} \max _{M_{i}, \Sigma_{i}}\left\{\log \mathcal{L}\left(M_{i}, \Sigma_{i}\right)-p\left[\alpha e_{i}^{T}\left(M_{i}\right)+(1-\alpha) e_{i}^{F}\left(M_{i}\right)\right]\right\},
$$

where $\alpha \in[0,1]$ is a balancing coefficient and $p$ specifies the relative weight of the penalties.

The solution to pMLE offers a balance among the likelihood estimator and the errors in both time and frequency domains. When $p$ is small, the objective is near the optimal $\log \mathcal{L}\left(M_{i}, \Sigma_{i}\right)$, which provides a relatively good initial solution. Then, as $p$ increases, the errors in both domains are reduced significantly, while sacrificing the likelihood. Toward the end of the iteration, when either $e_{i}^{F}$ or $e_{i}^{T}$ is near its local or global minimum, the increasing $p$ can still guarantee the reduction of the other error. The objective (6) is expected to finally achieve the best value when no further improvement is possible.

To solve pMLE at each iteration, any meta-heuristic search method that allows embedded simulation could be effectively used. We use a genetic algorithm that is terminated when either the number of iterations reaching a set maximum or the fit of the model does not improve over a number of iterations.

\section{Describing Function Approach Prediction}

In the previous section, frequency-domain feedback is provided via spectrum analysis of the simulated trajectories. An alternative approach could be developed based on analytical predications from so as to reduce computational burden. Given the oscillation properties of the leading vehicle, the DFA can be used to predict those of the following vehicle under a class of nonlinear car-following law $M_{i}$ that governs the oscillatory components of two adjacent trajectories:

$$
x_{i}^{o}(t)=\mathbf{L}_{i}\left[\mathbf{N}_{i}\left(x_{i-1}^{o}(t)-x_{i}^{o}(t)\right)\right]
$$

where $\mathbf{L}_{i}[\cdot]$ is a linear operator with low-pass filter (e.g., integrator) and $\mathbf{N}_{i}(\cdot)$ is a nonlinear function of spacing, in which the output retains the same fundamental frequency as the input. 
Place Figure 2 about here.

We reformulate $(7)$ in the frequency domain. Let $X_{i-1}(\Omega), X_{i}(\Omega)$, and $\widetilde{\mathbf{L}}_{i}(\Omega)$ be the Fourier transforms ${ }^{2}$ of $x_{i-1}^{o}(t), x_{i}^{o}(t)$, and $\mathbf{L}_{i}[\cdot]$, respectively, and $S_{i}(\Omega):=X_{i-1}(\Omega)-X_{i}(\Omega)$. Taking Fourier transform on both sides of (7) yields

$$
X_{i}(\Omega) \approx \tilde{\mathbf{L}}_{i}(\Omega) \cdot \tilde{\mathbf{N}}_{i}\left(\left|S_{i}(\Omega)\right|\right) \cdot S_{i}(\Omega),
$$

where

$$
\widetilde{\mathbf{N}}_{i}(A)=\frac{2 \int_{0}^{2 \pi} \mathbf{N}_{i}\left(A \sin (t) e^{-j t}\right) d t}{\pi}
$$

is the describing function.

Li et al. (2012) proves $\left|X_{i}(\Omega)\right|$ can be predicted by solving the following system of equations,

$$
\left\{\begin{array}{l}
\left|X_{i}(\Omega)\right|=\left|\widetilde{\mathbf{L}}_{i}(\Omega) \cdot \widetilde{\mathbf{N}}_{i}\left(\left|S_{i}(\Omega)\right|\right)\right| \cdot\left|S_{i}(\Omega)\right| \\
\left|X_{i-1}(\Omega)\right|=\left|1+\widetilde{\mathbf{L}}_{i}(\Omega) \cdot \widetilde{\mathbf{N}}_{i}\left(\left|S_{i}(\Omega)\right|\right)\right| \cdot\left|S_{i}(\Omega)\right|
\end{array}\right.
$$

Denote the solution of (9) by

$$
\left|X_{i}(\Omega)\right|=\operatorname{DFA}_{M_{i}}\left(\left|X_{i-1}(\Omega)\right|, \Omega\right) .
$$

Now, we are able to address the oscillation propagation reproduction. We first use the wavelet method described in section 2.2.2 to obtain the oscillation properties of the whole platoon, $\left\{\Omega_{i}\right\}_{i=0}^{n}$ and $\left\{A_{i}\right\}_{i=0}^{n}$. We then define the amplification ratio of vehicle $i$ as $R_{i}=A_{i-1} / A_{i}$ for $i=1,2, \ldots, n$, which is an important factor depicting the oscillation propagation.

Theoretically, $\Omega_{i-1}=\Omega_{i}$ should always hold due to the assumption of $\mathbf{N}_{i}(\cdot)$. There may, however, exist small gaps between them from the above wavelet method. Here we propose a simple modification: (i) combine trajectories $\left\{\mathbf{x}_{i-1}, \mathbf{x}_{i}\right\}$ into one single trajectory $\mathbf{x}_{i-1, i}$ by shifting the first point of $\mathbf{x}_{i}$ to the last point of $\mathbf{x}_{i-1}$ in the time-space diagram; (ii) repeat the above wavelet method to $\mathbf{x}_{i-1, i}$ to obtain the fundamental frequency, denoted by $\bar{\Omega}_{i}$; (iii) adjust both $\Omega_{i-1}$ and $\Omega_{i}$ to $\bar{\Omega}_{i}$. Note that this modification is conducted for each pair $\left\{\mathbf{x}_{i-1}, \mathbf{x}_{i}\right\}$ and $\bar{\Omega}_{i}$ is allowed to vary over $i$.

Then our task is to estimate $\hat{A}_{i}^{\prime}$ based on $A_{i-1}, \bar{\Omega}_{i}$, and the calibrated parameters $M_{i}$. This can be obtained from (10), i.e.,

$$
\hat{A}_{i}^{\prime}=\operatorname{DFA}_{M_{i}}\left(A_{i-1}, \bar{\Omega}_{i}\right) .
$$

This term $\hat{A}_{i}^{\prime}$ can be considered as an analytical counterpart of, and can be used to estimate, $\hat{A}_{i}$ in (5).

\section{Numerical Example}

In this section, empirical trajectory data are used to validate the proposed model calibration and oscillation prediction framework. For illustration, we consider two vehicle platoons from the NGSIM dataset, which were recorded from southbound US 101 in Los Angeles, CA from 7:50 - 8:35 AM on June 15, 2005.

Place Figure 3 about here.

\footnotetext{
${ }^{2}$ The Fourier transform of $f(t)$ is $F(\Omega)=\int_{-\infty}^{\infty} f(t) e^{-j \Omega t} d t$.
} 
We assume that the drivers follow the Newell's nonlinear car-following law (Newell, 1961), as shown in Figure 3. The following vehicle $i$ adjusts its desired velocity $v_{i}^{*}(t)$ linearly based on the spacing to its leading vehicle $i-1$, subject to physical speed bounds; i.e., in (1) we let

Newell's Model:

$$
v_{i}^{*}(t)=\operatorname{mid}\left\{0, k_{i}\left[x_{i-1}\left(t-\tau_{i}\right)-x_{i}\left(t-\tau_{i}\right)\right]-\omega_{i}, v_{i}^{\max }\right\}, \forall t \in T_{i}
$$

where $\omega_{i}$ is the backward shockwave speed, $k_{i}$ is the sensitivity factor of driving aggressiveness, $v_{i}^{\max }$ is the upper bound on velocity, and again, $\tau_{i}$ is the reaction time. The car-following behavior of vehicle $i$ can be fully specified by $M_{i}:=\left\{k_{i}, \tau_{i}, \omega_{i}, v_{i}^{\max }\right\}$. For convenience, we further define the stopping distance as $s_{i}^{0}:=\omega_{i} / k_{i}$ and the spacing when desired velocity first reaches the upper bound as $s_{i}^{\max }:=\left(\omega_{i}+v_{i}^{\max }\right) / k_{i}$.

For most observations, we assume a normal distribution for the error term, with zero mean and standard deviation $\sigma_{i}$, hence we simply have $\Sigma_{i}=\left\{\sigma_{i}\right\}$. Since drivers do not drive backwards, however, the folded normal distribution is used instead for the case when $v_{i}^{*}(t)=0$. Define $I(\cdot)=1$ if the expression in $(\cdot)$ is true; or 0 otherwise. Then the likelihood function for a given pair of observed trajectories $\left\{\mathbf{x}_{i-1}, \mathbf{x}_{i}\right\}$ is given by

$\mathcal{L}\left(M_{i}, \sigma_{i}\right)=\prod_{t \in T_{i}}\left[\frac{1}{\sigma_{i}} \tilde{\phi}\left(\frac{v_{i}(t)}{\sigma_{i}}\right)\right]^{I\left(v_{i}^{*}(t)=0\right)} \cdot\left[\frac{1}{\sigma_{i}} \phi\left(\frac{v_{i}(t)-v_{i}^{\max }(t)}{\sigma_{i}}\right)\right]^{I\left(s_{i}\left(t, \tau_{i}\right) \geq s_{i}^{\max }\right)} \cdot\left[\frac{1}{\sigma_{i}} \phi\left(\frac{v_{i}(t)-v_{i}^{*}(t)}{\sigma_{i}}\right)\right]^{I\left(s_{i}\left(t, \tau_{i}\right)<s_{i}^{\max }\right) \cdot I\left(v_{i}^{*}(t) \geq 0\right)}$,

where $\tilde{\phi}(\cdot)$ and $\phi(\cdot)$ now are the probability density functions of the folded and the normal distribution, respectively.

\subsection{Case I}

We will first illustrate the proposed model calibration technique using a platoon consisting of Vehicles 81 to 93 in the NGSIM data. We set $\alpha=0.5$ for each pair of vehicle trajectories and use the genetic algorithm to find the model and probability parameters. We linearly increase $p$ over the number of generations. Such heuristic parameter settings can provide reasonable calibration results in general. We leave more complicated balancing and updating scheme for future study. The calibrated parameters are listed in Table 1.

Place Table 1 about here.

Place Table 2 about here.

Place Figure 4 about here.

In Table 1, column " $-\log \mathcal{L}$ " indicates the negative $\log$ likelihood at convergence, while $e^{F}$ and $e^{T}$ respectively indicate the residual frequency- and time-domain errors. Rows "Avg." and "CV" show the mean and coefficient of variation (i.e., the standard deviation divided by the mean) of the entire platoon, respectively. Table 2 shows the minimum, maximum, and average frequencyand time-domain errors for 10 runs of the genetic algorithm. We observe that the majority of the parameters have reasonable values, although there is obvious heterogeneity across the drivers. The frequency-domain errors are much smaller than the time-domain errors for most vehicles, implying far better reproduction in the frequency domain. Towards the end of the iterative calibration algorithm, it is often observed that the time-domain error will remain relatively stable, while the frequency-domain error continues to decrease to an infinitesimal value. 
Figure 4 offers a closer look at the velocity-spacing diagrams of Vehicles 88 and 92, as examples. For Vehicle 88, the observed data are distributed across the linear and truncation segments of the Newell's model. The observed data for Vehicle 92 are more clustered within the linear segment in this case, the parameter value $v^{\max }$ seems to have little effect on the time-domain reproduction, as will be seen later in the sensitivity analysis.

Figures 5 illustrates the reproduction of the field data based on the calibrated car-following models. For the time domain, Figure 5(a) shows that the field and simulated trajectories match very well for the whole platoon ${ }^{3}$. Meanwhile, for the frequency domain, Figure 5(b) shows the reproduced oscillation propagation as indicated by, $A_{i}$, the cumulative amplitude growth from the first vehicle $(\# 81)$, and $R_{i}$, the pair-wise amplitude growth. The reproduction in the frequency domain is shown using both the simulation-based method (based on $\hat{A}_{i}$ in Section 2.2.2) and the DFA (based on $\hat{A}_{i}^{\prime}$ in Section 3), for comparison. It should be noted that the frequency-domain feedback used in the calibration for these numerical examples is found using the spectrum analysis of the simulated trajectories, not the DFA. The DFA results here use the calibrated model to predict the oscillations. We observe that they both match their corresponding field measurements very well, which simultaneously validates both the analytical prediction and the simulation in the frequency domain, verifying that either method could be used to provide the frequency-domain feedback.

Place Figure 5 about here.

\subsection{Case II}

We next repeat the same calibration procedure for another platoon consisting of Vehicles 311 to 320 from the same NGSIM data - note that now there are two oscillating cycles in the data. The calibrated parameters for the entire platoon are listed in Table 3. It can be seen that, as in Case I, most parameter values seem reasonable, heterogeneity seems to exist across drivers, and the frequency-domain errors are extremely small. Table 4 shows the minimum, maximum, and average time- and frequency-domain errors for 10 runs.

Place Table 3 about here.

Place Table 4 about here.

Figure 6 again shows that the proposed calibration method yields reasonable results in both the time and frequency domains.

Place Figure 6 about here.

\subsection{Sensitivity Analysis}

Now, we will draw additional insights by conducting a series of sensitivity analyses on the platoon from Case I. First, we evaluate how the value of $\alpha$ (i.e., relative weights of time- and frequencydomain error feedback) affect the calibration results from (3). We compare three scenarios: (i) $\alpha=1$; i.e., the frequency-domain penalties are not considered, (ii) $\alpha=0$; i.e., the time-domain penalties are not considered, and (iii) $p=0$; i.e., both time- and frequency-domain penalties are not considered.

\footnotetext{
${ }^{3}$ Here, every following vehicle trajectory is reproduced based on its observed (i.e., accurate) leading vehicle trajectory, because this is how our model calibration feedback is defined. There may be cumulative reproduction errors in a platoon if we instead use the reproduced leading vehicle trajectory to reproduce the next following vehicle trajectory. This error could be mitigated by directly defining the time-domain feedback error as such cumulative errors. Section 5 has more discussion on this issue.
} 
Place Figure 7 about here.

Figure 7 shows the reproduction of traffic based on calibration results for each of the three scenarios. We can clearly observe that when $\alpha=1$, the reproduction of oscillation propagation in Figures 7(b) is much worse than that in Figure 5(b). It should be noted here that the time-domain error for this scenario is relatively small, while the frequency-domain error is very large. There is a good fit in the time-space trajectories, but the oscillation prediction is not accurate at all. The frequency-domain error only reflects the oscillatory part of the trajectory, while the time-domain error reflects the overall accuracy of the entire trajectory. In this scenario, the oscillatory part of the trajectory is not reproduced as well as the rest of the trajectory. Such discordance is not unexpected, especially for those lower order car-following laws. For example, oscillation can never be amplified based on the simplified Newell car-following model (Newell, 2002) regardless of the parameters. This is the reason why modifications had to be added to this model in order to allow oscillation to occur (e.g., Chen et al., 2012; Laval et al., 2014). On the contrary, when $\alpha=0$, the reproduction of time-domain trajectories in Figures 7(c) becomes worse than that in Figure 5(a). Note that some simulated vehicle trajectories even contain artificial oscillations due to the lack of control of time-domain properties. In addition, when neither time- nor frequency-domain errors are considered the reproductions in both domains are much worse than those in Scenarios (i) and (ii).

Second, we explore how sensitive time- and frequency-domain errors are to each model parameter near optimum. This test indirectly report efficiency of the parameter estimators. For illustration, for each Vehicle $i$, we recalculate $e_{i}^{F}$ and $e_{i}^{T}$ when $k_{i}, \tau_{i}, \omega_{i}$, and $v_{i}^{\max }$ are respectively set, one at a time, to be either $10 \%$ larger or $10 \%$ smaller than its corresponding calibrated value in Case I. The results are summarized in Table 5.

Place Table 5 about here.

Several trends can be seen here. First, the absolute changes of $e^{F}$ are greater than that of $e^{T}$ when any of $k, \tau$, and $v^{\max }$ varies. On the contrary, the variation of $w$ only affects $e^{T}$, and the variation of $\sigma$ has no effect on either $e^{F}$ or $e^{T}$. These results imply that $k, \tau$, and $v^{\max }$ jointly determine the oscillatory properties of vehicle trajectories, while the time-domain trajectory properties depends also on $\omega$. In addition, no matter $k$ increases or decreases, both $e^{F}$ and $e^{T}$ increase by a relatively large amount, implying that $k$ is a critical factor that significantly reduces traffic reproduction in both time and frequency domains.

Finally, we conduct a parsimony test on the model parameters to see if some of them can share the same values across the entire platoon, yet without significantly increasing the calibration errors. It is straightforward that $\sigma$ could be set to the same value across drivers, based on the sensitivity results in Table 5 above. Noting that $k$ and $v^{\max }$ have relatively small CV (see the last row in Table 1 ), we assume they are also likely to be the same across drivers. Let $\bar{\sigma}, \bar{k}$, and $\bar{v}^{\text {max }}$ be the mean value of $\sigma_{i}, k_{i}$, and $v_{i}^{\max }$ across the entire platoon, respectively. Then, we repeat the calibration on Case I using fixed values of $k_{i}=\bar{k}, \sigma_{i}=\bar{\sigma}$, and $v_{i}^{\max }=\bar{v}^{\max }$ for all $i$, and only calibrate $\tau_{i}$ and $\omega_{i}$. The results are listed in Table 6, and the reproduction of traffic properties are shown in Figure 8.

Place Table 6 about here.

Place Figure 8 about here.

This result shows that it is fairly reasonable to restrict $\sigma, k$, and $v^{\max }$ to be the same across the drivers in this platoon. This restriction leads to slightly larger errors in the time and frequency domains, as expected due to the reduced degrees of freedom, but the calibrated model still performs reasonably well. 


\section{Conclusion and Discussion}

Traffic oscillation is commonly seen in congested traffic to cause various types of inefficiencies. In order to mitigate its negative effects, we need a better understanding of its mechanism. Thus, various car-following laws have been proposed to describe how drivers behave in congestion and how such behavior may cause oscillations. There are still, however, discrepancies between theoretical predictions and field observations. Calibrated car-following laws are found to have difficulty reproducing field-observed or simulated traffic, especially when oscillation properties are used as a criterion.

We propose a systematic approach to calibrate nonlinear car-following laws. The method uses a combination of techniques including maximum likelihood estimation and time- and frequencydomain prediction error feedback. We perform model calibration on each pair of consecutive leadingfollowing vehicle trajectories in a platoon. Noises in the observation data due to errors in data collection or errors in modeling driver's behavior (a human does not always strictly follow a carfollowing law) make a perfect model impossible, but as shown by a series of numerical experiments on field data, our approach yields very promising results. The calibrated car-following model not only provides accurate time-space trajectories in the time domain but also predicts oscillation propagation precisely in the frequency domain.

One shall recall from Footnote 2 that we choose to perform the model calibration sequentially on each pair of trajectories. If we simulate all trajectories in a platoon using a different boundary condition (e.g., only the trajectory of the first vehicle of the platoon, the starting points of each following trajectory), then the trajectory reproduction tends to cumulate larger errors under the same calibrated car-following laws. This has been observed in our numerical experiments. However, it does not have to be the case. The parameters of all drivers in the platoon, i.e., $\left\{M_{1}, M_{2}, \ldots, M_{n}\right\}$ can instead be estimated simultaneously, and the reproduction errors of the trajectories can be defined for the entire platoon based on the new boundary condition (i.e., for every vehicle pair, use reproduced leading trajectory to simulate the following trajectory). Such an extension can be done without changing our proposed methodological framework, but requires a more complete trajectory input dataset. In particular, obtaining the integrated time-domain feedback, which requires the leading and the following vehicles' trajectories aligned with the same time frame, will cumulatively "cut" the length of the effective time horizon for trajectories in calibration. We will pursue further work in this direction in the near future.

There are also other parts of the current work that could be strengthened and explored through future study. For example, different nonlinear car-following laws besides Newell's model could be adopted to further demonstrate the applicability of the proposed framework. There is always a trade-off between the performance and simplicity of a model. In reality, driver behavior in congested traffic is complicated and interdependent. There are other, more complex, models developed recently that may more accurately reproduce a particular behavior and account for the interdependence seen in oscillating traffic. These models could be considered further, in a similar manner as the proposed framework, and may offer additional insights into the phenomenon. The technique could also be applied to other platoons or datasets to confirm the validity of the proposed technique. The calibrated models could be directly used as the foundation for further field studies, especially on predicting and mitigating traffic oscillations. More investigation could also focus on finding the key model parameters that have the biggest impact on the propagation of oscillations; such an understanding may lay the foundation for the development of strategies that can mitigate oscillations 
and their adverse effects. The examples in this paper used the genetic algorithm search algorithm to find the best parameters, but if a faster search algorithm is developed and used, then it may even be possible to run the calibration on a highway in real-time, allowing us to implement any mitigation strategies dynamically.

\section{Acknowledgment}

The authors would like to thank Profs. Michael H. Zhang (UC Davis) and Serge Hoogendoorn (TU Delft), and other participants at the Symposium Celebrating 50 Years of Traffic Flow Theory (Portland, August 2014) for providing very helpful comments during a symposium presentation. This research was supported in part by the U.S. National Science Foundation via Grant CMMI0748067.

\section{References}

Bando, M., Hasebe, K., Nakayama, A., Shibata, A., Sugiyama, Y., 1995. Dynamical model of traffic congestion and numerical simulation. Physical Review E 51 (2), 1035.

Chandler, R. E., Herman, R., Montroll, E. W., 1958. Traffic dynamics: studies in car following. Operations research $6(2), 165-184$.

Chen, D., Laval, J., Zheng, Z., Ahn, S., 2012. A behavioral car-following model that captures traffic oscillations. Transportation research part B 46 (6), 744-761.

Ciuffo, B. F., Punzo, V., Torrieri, V., 2007. A framework for the calibration of microscopic traffic flow models. In: Transportation Research Board 86th Annual Meeting. No. 07-0106.

Daubechies, I., 1992. Ten lectures on wavelets. Vol. 61. SIAM.

Ferrari, P., 1989. The effect of driver behaviour on motorway reliability. Transportation Research Part B: Methodological 23 (2), 139-150.

Gazis, D. C., Herman, R., Rothery, R. W., 1961. Nonlinear follow-the-leader models of traffic flow. Operations Research 9 (4), 545-567.

Gipps, P. G., 1981. A behavioural car-following model for computer simulation. Transportation Research Part B: Methodological 15 (2), 105-111.

Herman, R., Montroll, E. W., Potts, R. B., Rothery, R. W., 1959. Traffic dynamics: analysis of stability in car following. Operations research 7 (1), 86-106.

Kesting, A., Treiber, M., 2008. Calibrating car-following models by using trajectory data: Methodological study. Transportation Research Record: Journal of the Transportation Research Board 2088 (1), 148-156.

Koshi, M., Iwasaki, M., Ohkura, I., 1983. Some findings and an overview on vehicular flow characteristics. In: Proceedings of the 8th International Symposium on Transportation and Traffic Flow Theory. Vol. 198. University of Toronto: Toronto, Ontario, pp. 403-426. 
Kuhne, R., 1987. Freeway speed distribution and acceleration noise: calculations from a stochastic continuum theory and comparision with measurements. Transportation and Traffic Theory .

Laval, J. A., 2011. Hysteresis in traffic flow revisited: an improved measurement method. Transportation Research Part B: Methodological 45 (2), 385-391.

Laval, J. A., Toth, C. S., Zhou, Y., 2014. A parsimonious model for the formation of oscillations in car-following models. Transportation Research Part B 70, 228-238.

Li, X., Ouyang, Y., 2011. Characterization of traffic oscillation propagation under nonlinear carfollowing laws. Transportation research part B: methodological 45 (9), 1346-1361.

Li, X., Peng, F., Ouyang, Y., 2010. Measurement and estimation of traffic oscillation properties. Transportation Research Part B: Methodological 44 (1), 1-14.

Li, X., Wang, X., Ouyang, Y., 2012. Prediction and field validation of traffic oscillation propagation under nonlinear car-following laws. Transportation research part B: methodological 46 (3), 409423.

Neubert, L., Santen, L., Schadschneider, A., Schreckenberg, M., 1999. Single-vehicle data of highway traffic: A statistical analysis. Physical Review E 60 (6), 6480.

Newell, G. F., 1961. Nonlinear effects in the dynamics of car following. Operations Research 9 (2), 209-229.

Newell, G. F., 2002. A simplified car-following theory: a lower order model. Transportation Research Part B 36 (3), 195-205.

Treiber, M., Helbing, D., 2002. Reconstructing the spatio-temporal traffic dynamics from stationary detector data. Cooperative Transportation Dyamics 1 (3), 3-1.

Treiber, M., Hennecke, A., Helbing, D., 2000. Congested traffic states in empirical observations and microscopic simulations. Physical Review E 62 (2), 1805.

Treiber, M., Kesting, A., 2012. Validation of traffic flow models with respect to the spatiotemporal evolution of congested traffic patterns. Transportation research part C: emerging technologies 21 (1), 31-41.

Yeniay, O., 2005. Penalty function methods for constrained optimization with genetic algorithms. Mathematical and Computational Applications 10 (1), 45-56.

Zhao, T., Nie, Y. M., Zhang, Y., 2014. Extended spectral envelope method for detecting and analyzing traffic oscillations. Transportation Research Part B: Methodological 61, 1-16.

Zheng, Z., Ahn, S., Chen, D., Laval, J., 2011. Applications of wavelet transform for analysis of freeway traffic: Bottlenecks, transient traffic, and traffic oscillations. Transportation Research Part B: Methodological 45 (2), 372-384.

Zielke, B. A., Bertini, R. L., Treiber, M., 2008. Empirical measurement of freeway oscillation characteristics: an international comparison. Transportation Research Record: Journal of the Transportation Research Board 2088 (1), 57-67. 


\section{List of Figures}

1 Impact of reaction time on velocity-spacing plots (source: NGSIM data). $\ldots \ldots$. . 18

2 Block diagram for Car-Following Model . . . . . . . . . . . . . . . . . . . . 19

3 Newell's non-linear car-following law . . . . . . . . . . . . . . . . . . . 20

4 Velocity-spacing diagrams for calibrated Newell's models (left: vehicle 88, right: ve-

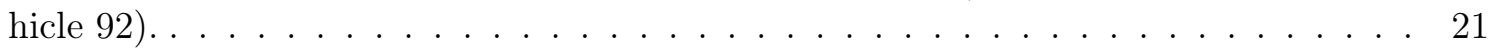

$5 \quad$ Reproduction result for Case I. (a) Time-space diagram; (b) Oscillation propagation. 22

6 Reproduction result for Case II. (a) Time-space diagram; (b) Oscillation propagation. 23

7 Reproduction result under different penalty schemes. (a) Time-space diagram $(\alpha=$ 1); (b) Oscillation propagation $(\alpha=1)$; (c) Time-space diagram $(\alpha=0)$; (d) Oscillation propagation $(\alpha=0)$; (e) Time-space diagram (no penalty); (f) Oscillation

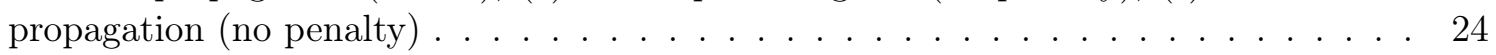

8 Reproduction result under parsimony. (a) Time-space diagram; (b) Oscillation prop-

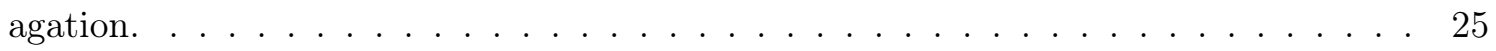




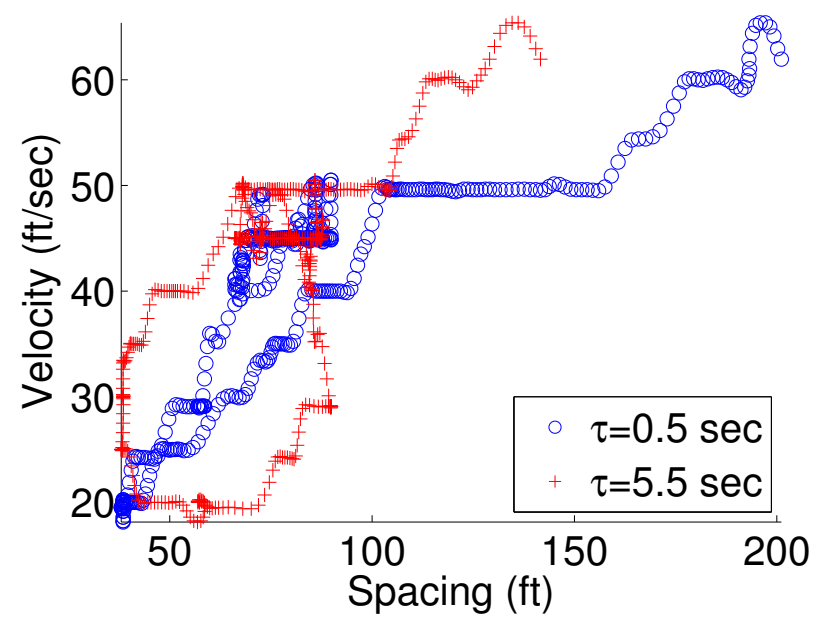

Figure 1: Impact of reaction time on velocity-spacing plots (source: NGSIM data). 


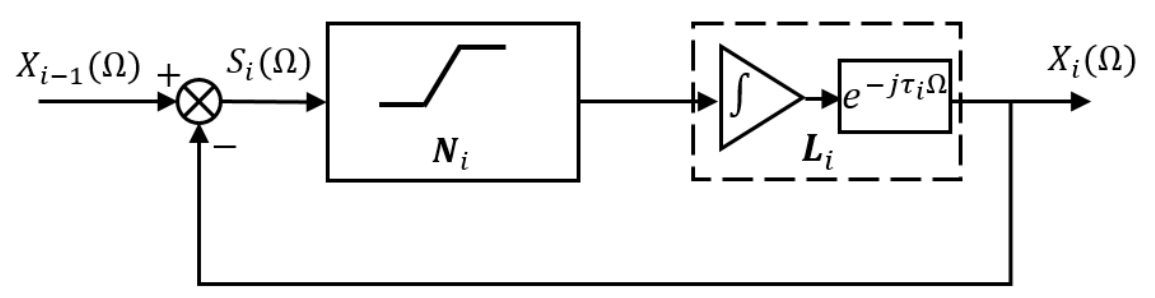

Figure 2: Block diagram for Car-Following Model 


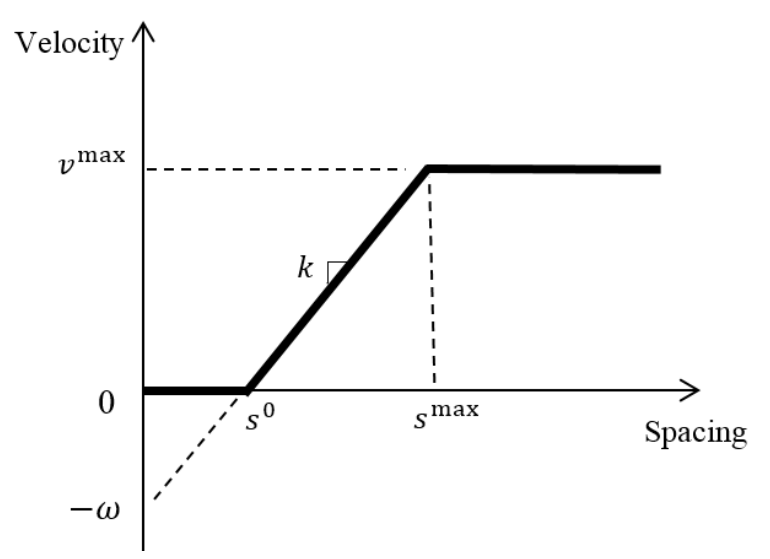

Figure 3: Newell's non-linear car-following law 

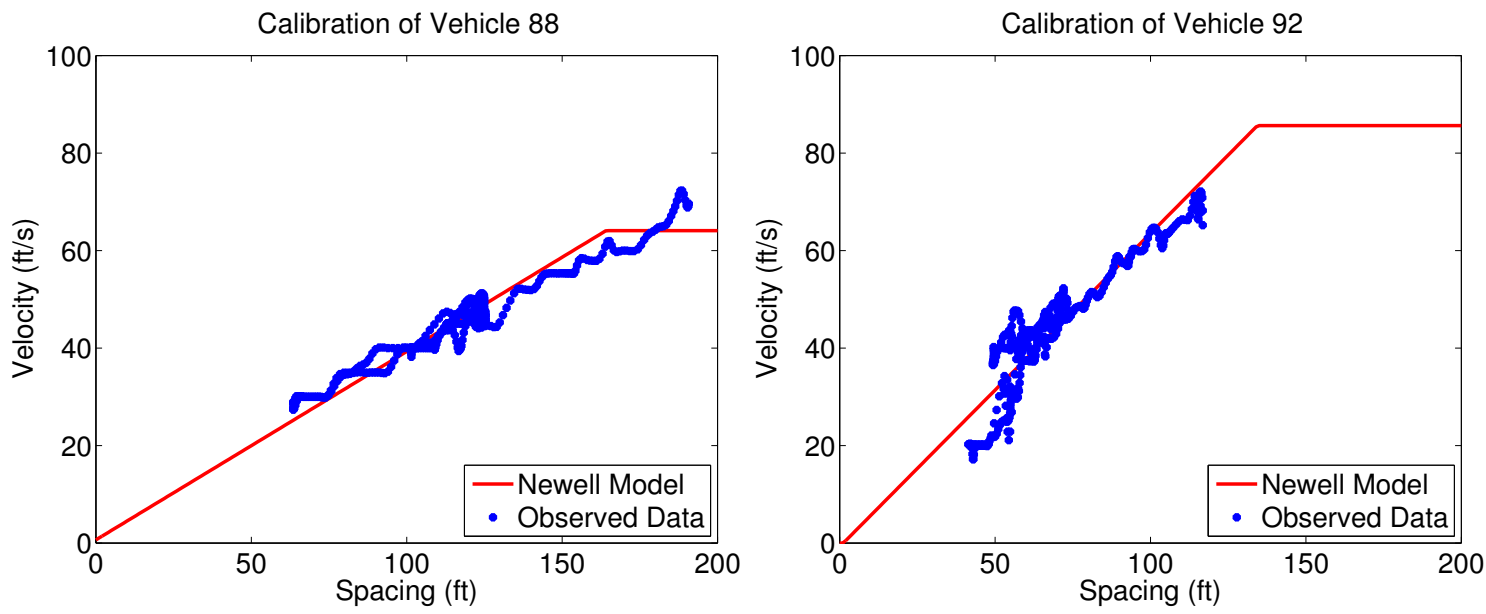

Figure 4: Velocity-spacing diagrams for calibrated Newell's models (left: vehicle 88, right: vehicle $92)$. 


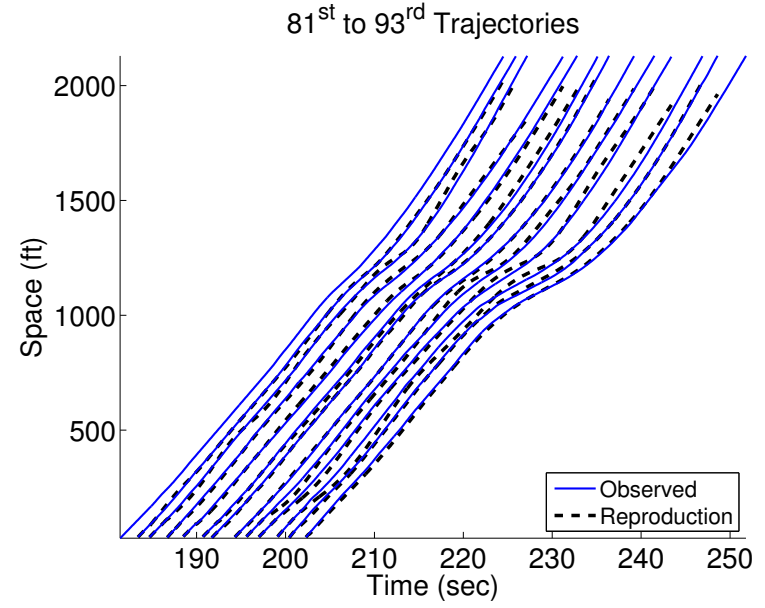

(a)
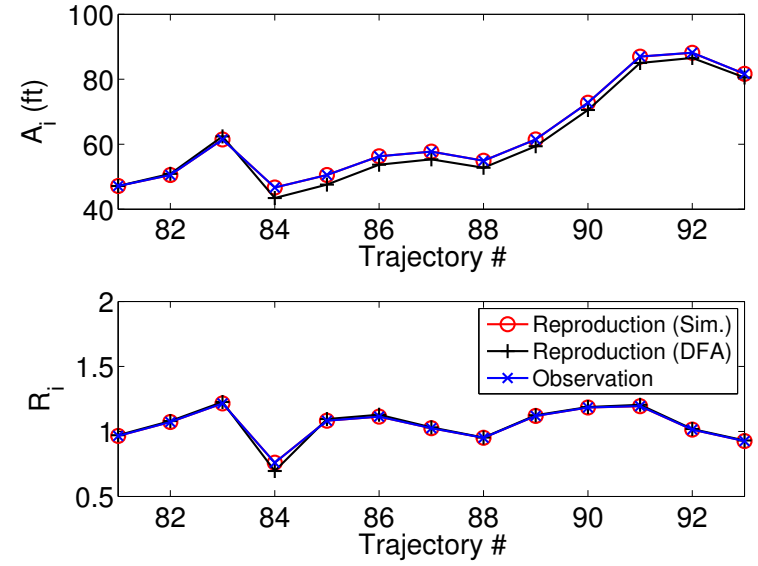

(b)

Figure 5: Reproduction result for Case I. (a) Time-space diagram; (b) Oscillation propagation. 


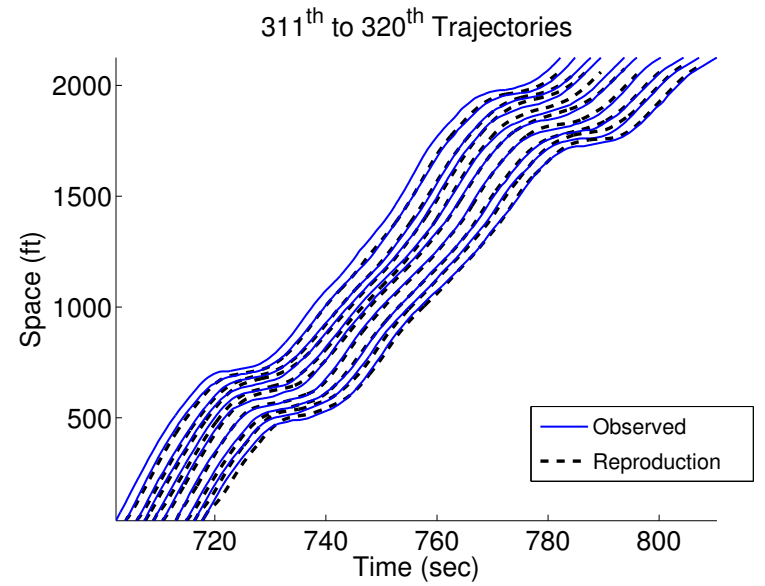

(a)
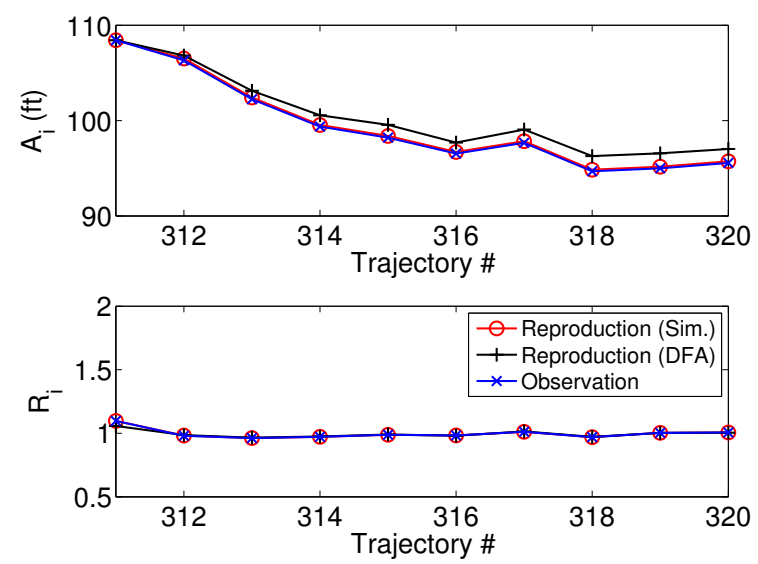

(b)

Figure 6: Reproduction result for Case II. (a) Time-space diagram; (b) Oscillation propagation. 


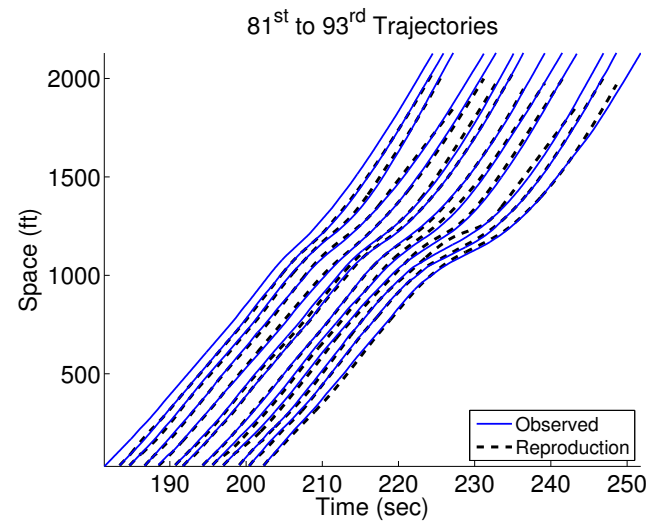

(a)

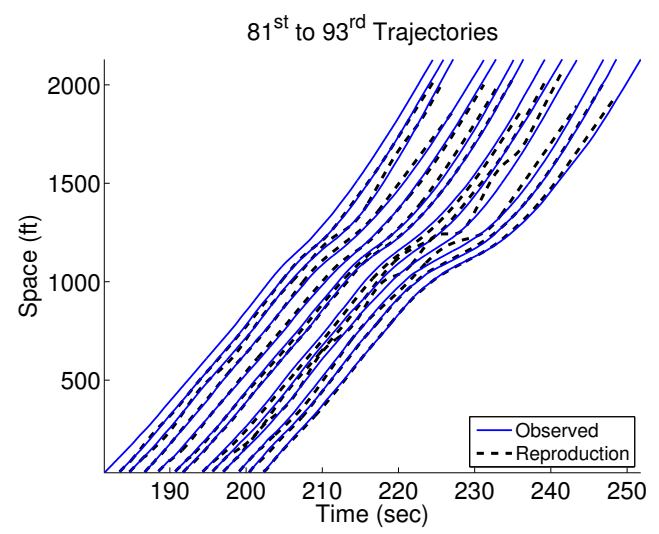

(c)

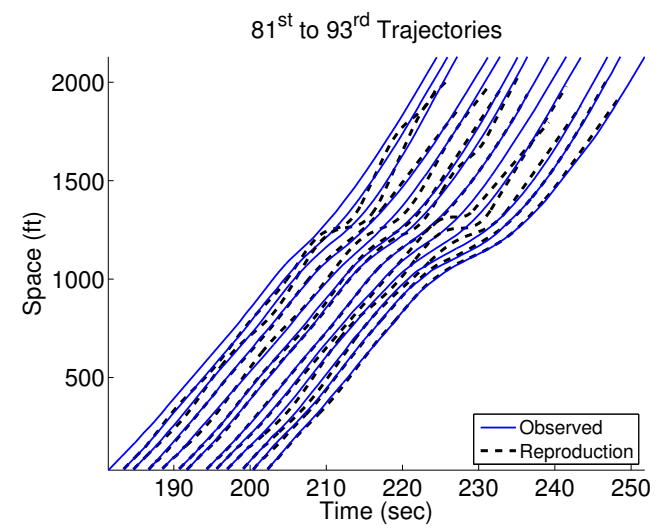

(e)
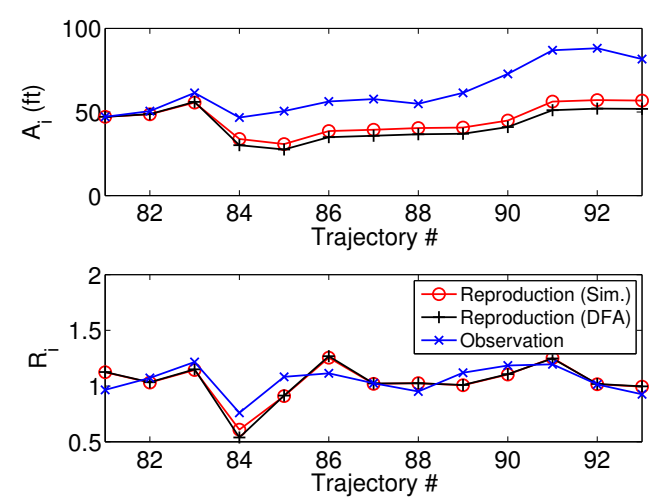

(b)
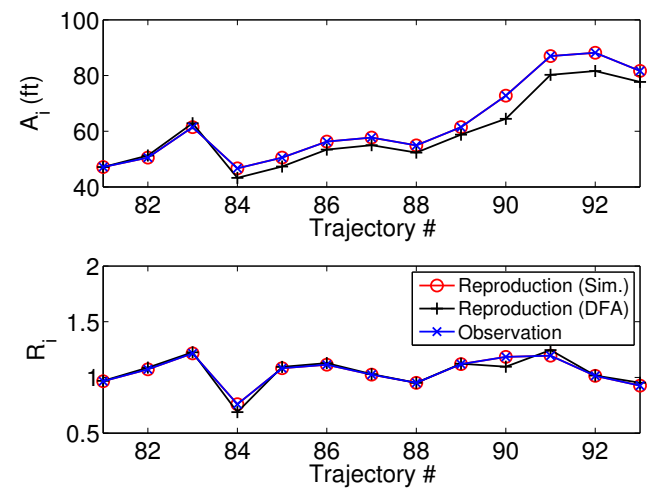

(d)
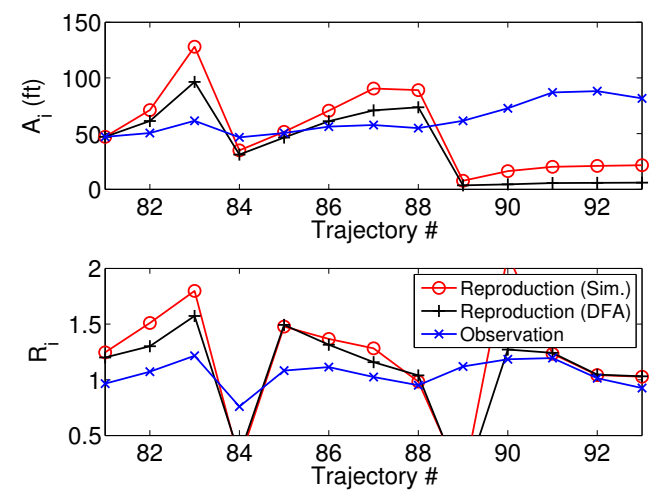

(f)

Figure 7: Reproduction result under different penalty schemes. (a) Time-space diagram $(\alpha=1)$; (b) Oscillation propagation $(\alpha=1)$; (c) Time-space diagram $(\alpha=0)$; (d) Oscillation propagation $(\alpha=0)$; (e) Time-space diagram (no penalty); (f) Oscillation propagation (no penalty) 


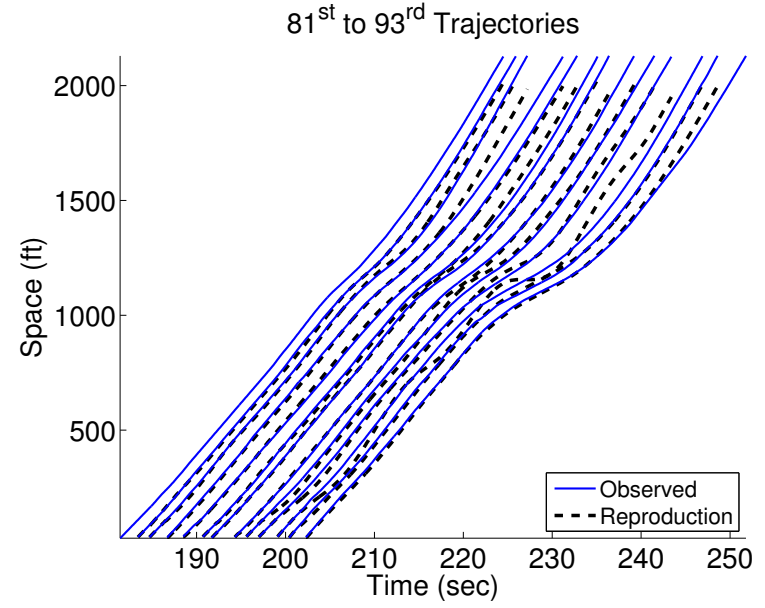

(a)
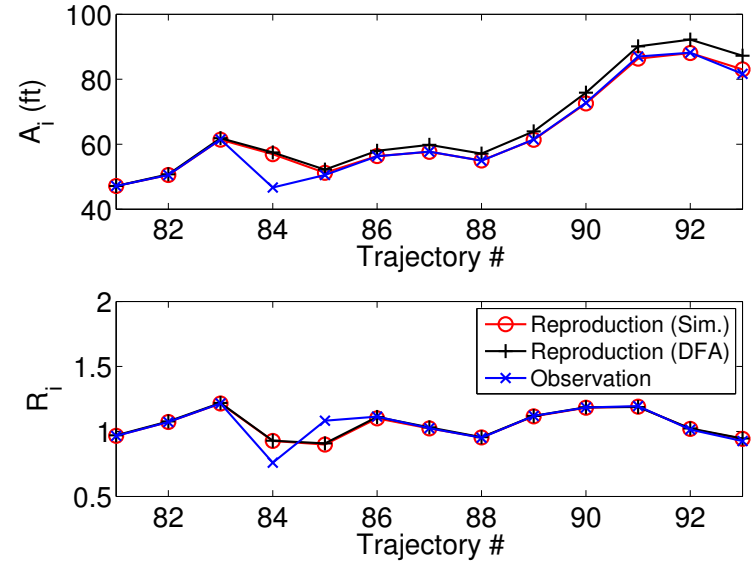

(b)

Figure 8: Reproduction result under parsimony. (a) Time-space diagram; (b) Oscillation propagation. 


\section{List of Tables}

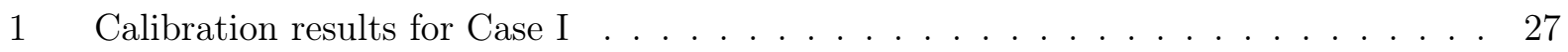

2 Minimum, Maximum, and Average Errors for Case I . . . . . . . . . . . . . 28

3 Calibration results for Case II . . . . . . . . . . . . . . . . . . . . . . . . . . . . 29

4 Minimum, Maximum, and Average Errors for Case II . . . . . . . . . . . . . . . 30

5 Sensitivity of reproduction errors to model parameters near optimum (e.g., $(0.2,-0.1)$

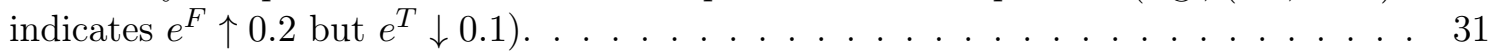

6 Calibration results of parsimony analysis . . . . . . . . . . . . 32 
Table 1: Calibration results for Case I

\begin{tabular}{ccccccccc}
\hline Vehicle & $k(1 / \mathrm{sec})$ & $\tau(\mathrm{sec})$ & $\omega(\mathrm{ft} / \mathrm{s})$ & $v^{\max }(\mathrm{ft} / \mathrm{s})$ & $\sigma(\mathrm{ft} / \mathrm{s})$ & $-\log \mathcal{L}$ & $e^{F}(\mathrm{ft})$ & $e^{T}(\mathrm{ft})$ \\
\hline 82 & 0.64 & 1.32 & 0.78 & 71.54 & 4.24 & 2.67 & $7.28 \mathrm{e}-6$ & 0.30 \\
83 & 0.74 & 2.07 & 2.00 & 67.94 & 6.27 & 3.09 & $8.84 \mathrm{e}-6$ & 0.76 \\
84 & 0.49 & 1.67 & -0.86 & 51.85 & 2.95 & 3.67 & $1.28 \mathrm{e}-6$ & 0.54 \\
85 & 0.54 & 1.52 & 6.37 & 77.95 & 7.32 & 3.43 & $2.86 \mathrm{e}-6$ & 0.76 \\
86 & 0.47 & 1.82 & 1.54 & 62.73 & 6.09 & 3.27 & $1.95 \mathrm{e}-5$ & 0.95 \\
87 & 0.91 & 0.92 & 2.75 & 84.31 & 4.37 & 2.83 & $1.67 \mathrm{e}-5$ & 0.24 \\
88 & 0.38 & 1.02 & -0.64 & 64.08 & 4.01 & 2.49 & $1.86 \mathrm{e}-5$ & 0.26 \\
89 & 0.63 & 2.02 & 3.05 & 61.97 & 6.94 & 3.67 & $2.15 \mathrm{e}-5$ & 0.89 \\
90 & 0.56 & 2.45 & 0.97 & 65.86 & 7.79 & 3.28 & $6.70 \mathrm{e}-5$ & 0.92 \\
91 & 0.41 & 2.67 & 0.31 & 59.00 & 4.92 & 4.56 & 0.033 & 1.56 \\
92 & 0.64 & 1.02 & 0.78 & 85.62 & 4.97 & 3.03 & 0.033 & 0.37 \\
93 & 0.42 & 0.42 & -5.05 & 60.88 & 5.71 & 3.11 & 0.031 & 0.75 \\
Avg. & 0.57 & 1.58 & 0.99 & 67.80 & 5.46 & 3.26 & 0.0027 & 0.69 \\
CV & 0.26 & 0.42 & 2.71 & 0.15 & 0.26 & 0.16 & 3.45 & 0.54 \\
\hline
\end{tabular}


Table 2: Minimum, Maximum, and Average Errors for Case I

\begin{tabular}{ccccccc}
\hline Vehicle & $\min e^{F}(\mathrm{ft})$ & $\max e^{F}(\mathrm{ft})$ & avg. $e^{F}(\mathrm{ft})$ & $\min e^{T}(\mathrm{ft})$ & $\max e^{T}(\mathrm{ft})$ & avg. $e^{T}(\mathrm{ft})$ \\
\hline 82 & $3.48 \mathrm{e}-8$ & $9.98 \mathrm{e}-6$ & $4.00 \mathrm{e}-6$ & 0.300 & 0.657 & 0.379 \\
83 & $2.36 \mathrm{e}-8$ & $3.24 \mathrm{e}-6$ & $7.75 \mathrm{e}-7$ & 0.652 & 1.406 & 0.770 \\
84 & $2.59 \mathrm{e}-7$ & $7.45 \mathrm{e}-5$ & $1.09 \mathrm{e}-5$ & 0.553 & 1.514 & 0.876 \\
85 & $8.29 \mathrm{e}-8$ & $2.95 \mathrm{e}-4$ & $3.48 \mathrm{e}-5$ & 0.761 & 1.154 & 0.881 \\
86 & $7.75 \mathrm{e}-10$ & $3.32 \mathrm{e}-5$ & $8.12 \mathrm{e}-6$ & 0.907 & 1.065 & 0.970 \\
87 & $4.13 \mathrm{e}-8$ & $3.75 \mathrm{e}-6$ & $9.62 \mathrm{e}-7$ & 0.221 & 0.313 & 0.241 \\
88 & $2.23 \mathrm{e}-7$ & $1.14 \mathrm{e}-4$ & $2.78 \mathrm{e}-5$ & 0.246 & 0.740 & 0.356 \\
89 & $2.33 \mathrm{e}-7$ & $3.27 \mathrm{e}-6$ & $9.16 \mathrm{e}-7$ & 0.888 & 1.031 & 0.950 \\
90 & $4.94 \mathrm{e}-8$ & $2.19 \mathrm{e}-5$ & $3.76 \mathrm{e}-6$ & 0.832 & 1.808 & 1.106 \\
91 & $2.13 \mathrm{e}-9$ & $3.42 \mathrm{e}-2$ & $1.02 \mathrm{e}-2$ & 1.419 & 2.243 & 1.819 \\
92 & $6.68 \mathrm{e}-8$ & $3.85 \mathrm{e}-5$ & $9.58 \mathrm{e}-6$ & 0.354 & 0.518 & 0.401 \\
93 & $5.00 \mathrm{e}-9$ & $9.52 \mathrm{e}-5$ & $1.02 \mathrm{e}-5$ & 0.459 & 0.838 & 0.665 \\
\hline
\end{tabular}


Table 3: Calibration results for Case II

\begin{tabular}{ccccccccc}
\hline Vehicle & $k(1 / \mathrm{sec})$ & $\tau(\mathrm{sec})$ & $\omega(\mathrm{ft} / \mathrm{s})$ & $v^{\max }(\mathrm{ft} / \mathrm{s})$ & $\sigma(\mathrm{ft} / \mathrm{s})$ & $-\log \mathcal{L}$ & $e^{F}(\mathrm{ft})$ & $e^{T}(\mathrm{ft})$ \\
\hline 312 & 0.53 & 0.52 & 6.93 & 59.05 & 8.87 & 3.29 & $1.20 \mathrm{e}-6$ & 0.41 \\
313 & 0.39 & 0.52 & 2.32 & 55.40 & 6.17 & 2.74 & $1.36 \mathrm{e}-5$ & 0.29 \\
314 & 0.56 & 0.12 & 0.77 & 89.85 & 3.80 & 3.52 & $5.78 \mathrm{e}-6$ & 0.48 \\
315 & 0.47 & 0.77 & 6.12 & 78.85 & 8.29 & 3.67 & $6.28 \mathrm{e}-6$ & 0.70 \\
316 & 0.50 & 0.41 & 1.45 & 44.52 & 9.90 & 3.42 & $5.82 \mathrm{e}-5$ & 0.48 \\
317 & 0.44 & 1.52 & 9.84 & 46.95 & 7.54 & 3.01 & $1.11 \mathrm{e}-5$ & 0.41 \\
318 & 0.40 & 0.42 & 6.16 & 46.10 & 5.35 & 3.02 & $3.20 \mathrm{e}-4$ & 0.36 \\
319 & 0.49 & 1.11 & 2.38 & 42.65 & 5.09 & 3.34 & $2.72 \mathrm{e}-4$ & 0.49 \\
320 & 0.42 & 1.35 & 1.61 & 45.89 & 7.44 & 3.23 & $1.17 \mathrm{e}-4$ & 0.51 \\
Avg. & 0.47 & 0.75 & 4.17 & 54.87 & 6.69 & 3.25 & $8.95 \mathrm{e}-5$ & 0.46 \\
CV & 0.12 & 0.63 & 0.75 & 0.31 & 0.27 & 0.09 & 1.37 & 0.24 \\
\hline
\end{tabular}


Table 4: Minimum, Maximum, and Average Errors for Case II

\begin{tabular}{ccccccc}
\hline Vehicle & $\min e^{F}(\mathrm{ft})$ & $\max e^{F}(\mathrm{ft})$ & avg. $e^{F}(\mathrm{ft})$ & $\min e^{T}(\mathrm{ft})$ & $\max e^{T}(\mathrm{ft})$ & avg. $e^{T}(\mathrm{ft})$ \\
\hline 312 & $6.51 \mathrm{e}-8$ & $7.63 \mathrm{e}-5$ & $1.10 \mathrm{e}-5$ & 0.399 & 0.738 & 0.507 \\
313 & $1.14 \mathrm{e}-7$ & $2.23 \mathrm{e}-4$ & $4.81 \mathrm{e}-5$ & 0.279 & 0.625 & 0.341 \\
314 & $7.56 \mathrm{e}-8$ & $2.46 \mathrm{e}-5$ & $9.69 \mathrm{e}-6$ & 0.482 & 1.181 & 0.599 \\
315 & $3.24 \mathrm{e}-8$ & $2.61 \mathrm{e}-5$ & $7.41 \mathrm{e}-6$ & 0.686 & 0.978 & 0.783 \\
316 & $1.90 \mathrm{e}-7$ & $5.68 \mathrm{e}-5$ & $1.42 \mathrm{e}-5$ & 0.365 & 0.639 & 0.462 \\
317 & $4.64 \mathrm{e}-7$ & $5.68 \mathrm{e}-4$ & $9.93 \mathrm{e}-5$ & 0.410 & 0.763 & 0.550 \\
318 & $1.90 \mathrm{e}-6$ & $1.03 \mathrm{e}-4$ & $3.12 \mathrm{e}-5$ & 0.364 & 0.536 & 0.424 \\
319 & $5.77 \mathrm{e}-8$ & $6.11 \mathrm{e}-5$ & $8.78 \mathrm{e}-6$ & 0.381 & 0.562 & 0.447 \\
320 & $5.82 \mathrm{e}-7$ & $2.61 \mathrm{e}-5$ & $7.56 \mathrm{e}-6$ & 0.432 & 0.683 & 0.506 \\
\hline
\end{tabular}


Table 5: Sensitivity of reproduction errors to model parameters near optimum (e.g., $(0.2,-0.1)$ indicates $e^{F} \uparrow 0.2$ but $\left.e^{T} \downarrow 0.1\right)$.

\begin{tabular}{|c|c|c|c|c|c|c|c|c|c|c|}
\hline Vehicle & $k \uparrow 10 \%$ & $k \downarrow 10 \%$ & $\tau \uparrow 10 \%$ & $\tau \downarrow 10 \%$ & $\omega \uparrow 10 \%$ & $\omega \downarrow 10 \%$ & $v^{\max } \uparrow 10 \%$ & $v^{\max } \downarrow 10 \%$ & $\sigma \uparrow 10 \%$ & $\sigma \downarrow 10 \%$ \\
\hline 82 & $(0.76,0.14)$ & $(1.14,0.25)$ & $(1.84,0.011)$ & $(1.06,-0.0033)$ & $(0,0.00050)$ & $(0,-0.00038)$ & $(0.17,0)$ & $(10.63,0.0052)$ & $(0,0)$ & $(0,0)$ \\
\hline 83 & $(2.68,0.15)$ & $(0.76,0038)$ & $(8.72,-0.020)$ & $(3.15,-0.048)$ & $(0,0.00021)$ & $(0,0.00021)$ & $(0.21,0.13)$ & $(12.97,-0.14)$ & $(0,0)$ & $(0,0)$ \\
\hline 84 & $(1.29,0.13)$ & $(0.93,0.19)$ & $(3.48,0.0027)$ & $(1.85,0.011)$ & $(0,0.00025)$ & $(0,-0.00010)$ & $(16.80,0.33)$ & $(36.23,0.35)$ & $(0,0)$ & $(0,0)$ \\
\hline 85 & $(1.70,0.08)$ & $(1.52,0.20)$ & $(5.13,0.037)$ & $(3.15,-0.016)$ & $(0,0.0022)$ & $(0,0.0022)$ & $(18.19,0)$ & $(39.22,0)$ & $(0,0)$ & $(0,0)$ \\
\hline 86 & $(2.26,0.16)$ & $(2.36,0.097)$ & $(7.93,-0.013)$ & $(4.83,0.016)$ & $(0,0.00014)$ & $(0,0.00014)$ & $(20.26,0.0056)$ & $(43.69,-0.098)$ & $(0,0)$ & $(0,0)$ \\
\hline 87 & $(2.41,0.11)$ & $(2.58,0.13)$ & $(8.67,0.0035)$ & $(5.17,-0.0012)$ & $(0,0.00045)$ & $(0,0.00044)$ & $(20.77,0)$ & $(44.79,0)$ & $(0,0)$ & $(0,0)$ \\
\hline 88 & $(3.55,0.36)$ & $(3.99,0.35)$ & $(9.76,-0.013)$ & $(5.68,0.014)$ & $(0,0.00083)$ & $(0,-0.00060)$ & $(19.77,0.018)$ & $(42.62,-0.0090)$ & $(0,0)$ & $(0,0)$ \\
\hline 89 & $(3.72,0.07)$ & $(4.31,0.076)$ & $(12.81,0.053)$ & $(7.45,-0.017)$ & $(0,-0.00015)$ & $(0,0.00084)$ & $(22.12,0.053)$ & $(47.70,0.014)$ & $(0,0)$ & $(0,0)$ \\
\hline 90 & $(3.84,0.09)$ & $(4.68,0.095)$ & $(17.89,0.18)$ & $(10.71,-0.060)$ & $\left(0,6.45 e^{-5}\right)$ & $\left(0,2.47 e^{-5}\right)$ & $\left(26.20,-2.29 e^{-5}\right)$ & $(56.48,-0.099)$ & $(0,0)$ & $(0,0)$ \\
\hline 91 & $(4.28,0.08)$ & $(5.71,0.12)$ & $(24.32,-0.092)$ & $(15.32,0.091)$ & $(0,0.00067)$ & $(0,-0.00066)$ & $(31.20,0.35)$ & $(67.47,-0.24)$ & $(0,0)$ & $(0,0)$ \\
\hline 92 & $(4.68,0.07)$ & $(6.27,0.17)$ & $(25.95,0.0031)$ & $(16.08,-0.0021)$ & $(0,0.000042)$ & $\left(0,4.17 e^{-5}\right)$ & $(31.63,0)$ & $(68.41,0)$ & $(0,0)$ & $(0,0)$ \\
\hline 93 & $(5.67,0.10)$ & $(7.34,0.084)$ & $(23.39,-0.0086)$ & $(14.89,0)$ & $(0,0.0028)$ & $(0,0.00097)$ & $(29.30,0.074)$ & $(63.36,-0.18)$ & $(0,0)$ & $(0,0)$ \\
\hline
\end{tabular}


Table 6: Calibration results of parsimony analysis

\begin{tabular}{|c|c|c|c|c|c|c|c|c|}
\hline Vehicle & $k(1 / \mathrm{sec})$ & $\tau(\mathrm{sec})$ & $\omega(\mathrm{ft} / \mathrm{s})$ & $v^{\max }(\mathrm{ft} / \mathrm{s})$ & $\sigma(\mathrm{ft} / \mathrm{s})$ & $-\log \mathcal{L}$ & $e^{F}(\mathrm{ft})$ & $e^{T}(\mathrm{ft})$ \\
\hline 82 & \multirow{12}{*}{0.57} & 1.35 & -1.11 & \multirow{12}{*}{67.81} & \multirow{12}{*}{5.47} & 3.07 & 0.07 & 0.49 \\
\hline 83 & & 1.94 & -7.98 & & & 2.94 & 0.01 & 0.56 \\
\hline 84 & & 0.12 & 8.21 & & & 3.97 & 10.3 & 1.81 \\
\hline 85 & & 0.12 & 10.2 & & & 3.68 & 0.74 & 0.58 \\
\hline 86 & & 1.67 & 9.17 & & & 3.67 & 0.13 & 1.13 \\
\hline 87 & & 1.12 & -15.7 & & & 2.83 & 0.02 & 0.35 \\
\hline 88 & & 0.48 & 20.92 & & & 3.29 & 0.14 & 0.65 \\
\hline 89 & & 1.95 & -0.45 & & & 3.99 & 0.02 & 0.90 \\
\hline 90 & & 2.45 & 1.77 & & & 3.49 & 0.14 & 0.93 \\
\hline 91 & & 2.74 & 12.57 & & & 5.13 & 0.49 & 2.28 \\
\hline 92 & & 1.49 & -1.53 & & & 3.14 & 0.03 & 0.49 \\
\hline 93 & & 0.13 & -5.81 & & & 3.63 & 1.48 & 1.01 \\
\hline
\end{tabular}

